\title{
Evaluation of left ventricular myocardial work quantitatively by pressure-strain loop in young strength athletes with different heart rates
}

Pengge $\mathrm{Li}^{1}$, Yonggao Zhang ${ }^{1}$, Lijin $\mathrm{Li}^{1}$, Mengjiao $\mathrm{Sun}^{1}$, Zhen $\mathrm{Li}^{1}$, Mengmeng $\mathrm{Liu}^{1}$, and Shaohua Hua ${ }^{1}$

${ }^{1}$ Zhengzhou University First Affiliated Hospital

October 29, 2021

\begin{abstract}
Objective: The present study aimed to investigate the difference in left ventricular (LV) global and regional myocardial (MW) of strength athletes with different heart rates (HR) through non-invasive LV pressure-strain loop (PSL) and further address the effect of athlete's heart rate variability on the LV systolic function. Methods: Fifty young professional wrestlers were collected randomly and divided into two groups in accordance with their different HR: the low HR (45 $\left.60 \mathrm{bpm}, n_{1}=25\right)$ and the high HR $\left(60 \sim 80 \mathrm{bpm}, n_{2}=25\right)$. Thirty individuals with gender- and age-matched healthy volunteers served as controls $\left(n_{3}=30\right)$. Global and regional MW parameters were evaluated using LV-PSL derived from speckle tracking echocardiography (STE) and brachial artery pressure, and then compared between the above three groups. Results: The indicators of global and regional MW did statistically significantly differ between the athlete and control groups. Peak strain dispersion (PSD) and global myocardial wasted work (GWW) increased while global myocardial work efficiency (GWE) reduced in LHR and HHR groups compared with the control group, and global myocardial work index (GWI), global myocardial constructive work (GCW), global longitudinal strain (GLS) decreased in LHR group ( $P_{i} 0.05$ ). In comparison to the LHR group, GWI, GCW, GWW, PSD increased in HHR group and GWE reduced ( $P ; 0.05)$. According to the regional MW analysis, the mean regional myocardial work index (RMWI) increasing gradually from basal to apical levels were similar across the three groups and regional myocardial work efficiency (RMWE) did not. Multiple linear regression analysis indicated that the HR, posterior wall thickness (PWT), interventricular septal thickness (IVST), GLS, and PSD were correlated with GWE ( $b^{\prime}=-0.247,-0.390,-0.370,0.340$, and -0.554 , respectively, $P \nmid 0.05)$. Conclusions: The LV contractile performance was more impaired in young strength athletes with high heart rates and PSL can be used to assess LV GMW and RMW quantitatively and accurately in reflecting LV systolic function.
\end{abstract}

\section{Hosted file}

Manuscript.docx available at https://authorea.com/users/443568/articles/543539-evaluationof-left-ventricular-myocardial-work-quantitatively-by-pressure-strain-loop-in-youngstrength-athletes-with-different-heart-rates

\section{Hosted file}

Figures.doc available at https://authorea.com/users/443568/articles/543539-evaluationof-left-ventricular-myocardial-work-quantitatively-by-pressure-strain-loop-in-youngstrength-athletes-with-different-heart-rates

\section{Hosted file}

Tables.doc available at https://authorea.com/users/443568/articles/543539-evaluationof-left-ventricular-myocardial-work-quantitatively-by-pressure-strain-loop-in-young- 
strength-athletes-with-different-heart-rates 